\title{
Rapid evaluation of particle properties using inverse SEM simulations
}

\author{
Kursat B. Bekar ${ }^{1}$, Thomas M. Miller, ${ }^{1, *}$,Bruce W. Patton ${ }^{1}$, and Charles F. Weber \\ ${ }^{1}$ Oak Ridge National Laboratory, Reactor and Nuclear Systems Division, P.O. Box 2008, Oak Ridge, Tennessee, 37831, \\ USA
}

\begin{abstract}
The characteristic X-rays produced by the interactions of the electron beam with the sample in a scanning electron microscope (SEM) are usually captured with a variable-energy detector, a process termed energy dispersive spectrometry (EDS). The purpose of this work is to exploit inverse simulations of SEM-EDS spectra to enable rapid determination of sample properties, particularly elemental composition. This is accomplished using penORNL, a modified version of PENELOPE, and a modified version of the traditional Levenberg-Marquardt nonlinear optimization algorithm, which together is referred to as MOZAIK-SEM. The overall conclusion of this work is that MOZAIK-SEM is a promising method for performing inverse analysis of X-ray spectra generated within a SEM. As this methodology exists now, MOZAIK-SEM has been shown to calculate the elemental composition of an unknown sample within a few percent of the actual composition.
\end{abstract}

\section{Introduction}

Analysis of small particles and material surfaces has progressed rapidly with the development of the scanning electron microscope (SEM) over the past half century. The characteristic X-rays produced by the interactions of the electron beam with the sample are usually captured with a variable-energy detector, a process termed energy dispersive spectrometry (EDS). Measuring the chemical composition and crystallographic phase of small samples using electrons and $\mathrm{X}$-rays in SEM instruments is termed electron probe microanalysis (EPMA). By focusing an electron beam to a diameter of a few nanometers and measuring X-rays fluoresced from the sample when it is struck by fast electrons, an analyst can determine the elements present (qualitative analysis) with a spatial resolution well below $1 \mu \mathrm{m}$. The problem of accurately measuring the relative elemental abundances (quantitative analysis) is more difficult and has only been conducted acceptably on samples that are flat, highly polished, and chemically uniform. When these conditions are met, the chemical composition can be measured with great precision by comparison with known standards that have been carefully prepared. However, most real-world environmental samples do not conform to these restrictions. Hence, it is desired to obtain both qualitative and quantitative analyses without using standards and without meticulous sample preparation.

The purpose of this work is to exploit inverse simulations of SEM-EDS spectra to enable rapid

\footnotetext{
${ }^{*}$ Corresponding author: millertm@ornl.gov

This manuscript has been authored by UT-Battelle, LLC, under Contract No. DE-AC0500OR22725 with the U.S. Department of Energy. The United States Government retains and the publisher, by accepting the article for publication, acknowledges that the United States Government retains a nonexclusive, paid-up, irrevocable, worldwide license to publish or
}

determination of sample properties, including both qualitative and quantitative chemical analyses. This requires a method to model the electron interactions with small samples that occur in an SEM and the subsequent generation of X-rays and an algorithm to minimize the difference between the measured and calculated SEM spectra by iteratively updating the elemental composition.

\section{2 penORNL}

The first task of this work was to evaluate the radiation transport codes available for modeling coupled electron / photon physics [1]. This survey showed that the Monte Carlo (MC) radiation transport code PENELOPE $[2,3]$ provided the best combination of accurate physics, variance reduction specifically tailored to the generation of characteristic X-rays, and speed on a single processor. A second code, MCNP6 [4], had the benefits of being a parallel code and including the ability to tally pulse heights, but was slower in execution on a single processor.

PENELOPE has only been developed as a serial code, since the primary focus of the developers is the electron/photon physics modeling. They assumed that most users would write their own main program or driver program. Because MC codes can achieve massive speedups using multiple processors, a new main program was written to create a parallel version of PENELOPE. This new parallel version of the code is named penORNL [5]. The development of penORNL created a code that had all the most important features needed: accurate

reproduce the published form of this manuscript, or allow others to do so, for United States Government purposes. The Department of Energy will provide public access to these results of federally sponsored research in accordance with the DOE Public Access Plan (http://energy.gov/downloads/doe-public-access-plan). 
physics, an efficient algorithm, variance reduction, parallel capability, and a preliminary implementation of pulse-height tallies.

\section{Detector response function}

To compare MC simulation results to experimental data from the SEM-EDS process, a detector response function is used to generate the detector pulse-height spectrum from the photon line spectrum calculated by the MC simulation of the X-rays incident on the detector. The detector response function is defined as the pulse-height distribution for any incident monoenergetic X-ray, indicated by $R\left(E^{\prime}, E\right)$, where $E^{\prime}$ is the incident photon energy and $E$ is the pulse-height energy. All our calculations used a typical $\mathrm{Si}(\mathrm{Li})$ detector response which was imported from the PENELOPE code package:

$$
R\left(E, E^{\prime}\right)=\frac{1}{\sigma \sqrt{2 \pi}} \exp \left[-\frac{\left(E-E^{\prime}\right)^{2}}{2 \sigma^{2}}\right],
$$

where $\sigma$ is the standard deviation of the photopeak and its relation to the full-width-at-half-maximum is given as $\mathrm{FWHM}=2.35 \sigma$. To get the relationship of detector photopeak standard deviation with respect to X-ray energy, a non-linear model is used:

$$
\sigma\left(E^{\prime}\right)=\sqrt{a_{1}+a_{2} E^{\prime}}
$$

A set of calculations was performed to optimize the coefficients $a_{1}$ and $a_{2}$ in the response function, to obtain better agreement between the spectra computed by penORNL and the measured spectra. Table 1 presents the optimal values of these coefficients.

Table 1. Coefficients used in the detector response function definition

\begin{tabular}{cc}
\hline Coefficient & Optimal value \\
\hline$a_{1}$ & 0.006179 \\
$a_{2}$ & $0.00255 / \mathrm{keV}$ \\
\hline
\end{tabular}

If $M(E)$ represents the $\mathrm{MC}$ line spectrum computed by penORNL, a realistic detector spectrum $C(E)$ can be calculated by convolution with the response function:

$$
C(E)=\int_{0}^{E_{\max }} R\left(E, E^{\prime}\right) M\left(E^{\prime}\right) d E^{\prime}
$$

In reality, discrete energy bins are used to describe detectors responses, and the continuous response function in Eq. (1) is replaced by the response matrix $\mathbf{R}=\left(r_{i j}\right)$, with elements $r_{i j}=R\left(E_{i}, E_{j}\right)$. The convolution integral in Eq. (3) is likewise replaced by the discretized form:

$$
C_{i}=C\left(E_{i}\right)=\sum_{j=1}^{N} R\left(E_{i}, E_{j}\right) \times M\left(E_{j}\right),
$$

which can be expressed in matrix form as

$$
C=\mathbf{R} M \text {, }
$$

where $M_{j}=M\left(E_{j}\right)$, and $M=\left(M_{1}, M_{2}, \ldots, M_{N}\right)^{\mathrm{T}}$ and $C=$ $\left(C_{1}, C_{2}, \ldots, C_{N}\right)^{\mathrm{T}}$ are vectors of computed line spectra and detector response spectra, respectively.

A measured SEM-EDS spectrum and a typical MC line spectrum computed by penORNL for the same standard (apatite) are shown in Figure 1(a). After the convolution operation was performed, the computed spectrum was represented in a more realistic shape, as presented in Figure 1(b). The difference (\%) shown in Figure 1(b) calculated between the measured spectrum and the convoluted spectrum is mostly due to improperly estimated bremsstrahlung background. Differences between the actual measurement and simulation can cause differences in the bremsstrahlung background; therefore, we observe such a difference after the convolution process.

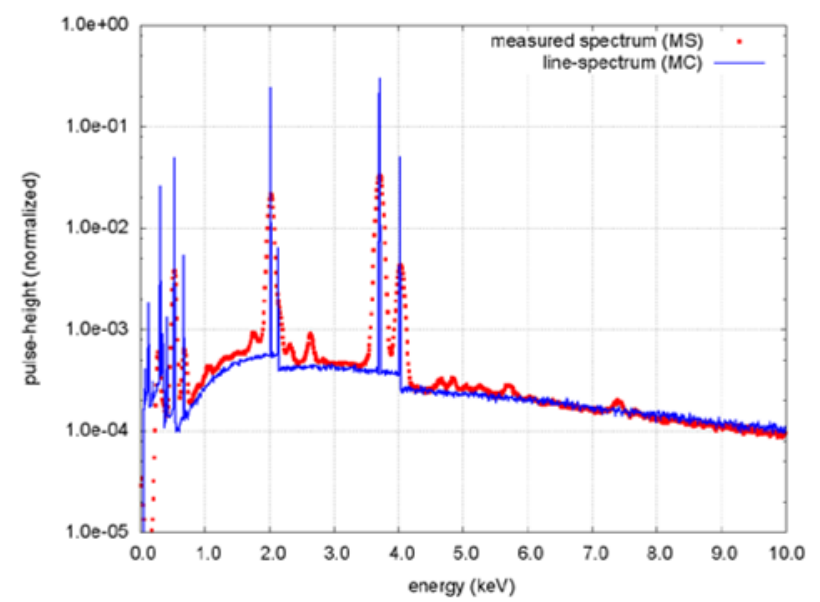

(a) Before convolution

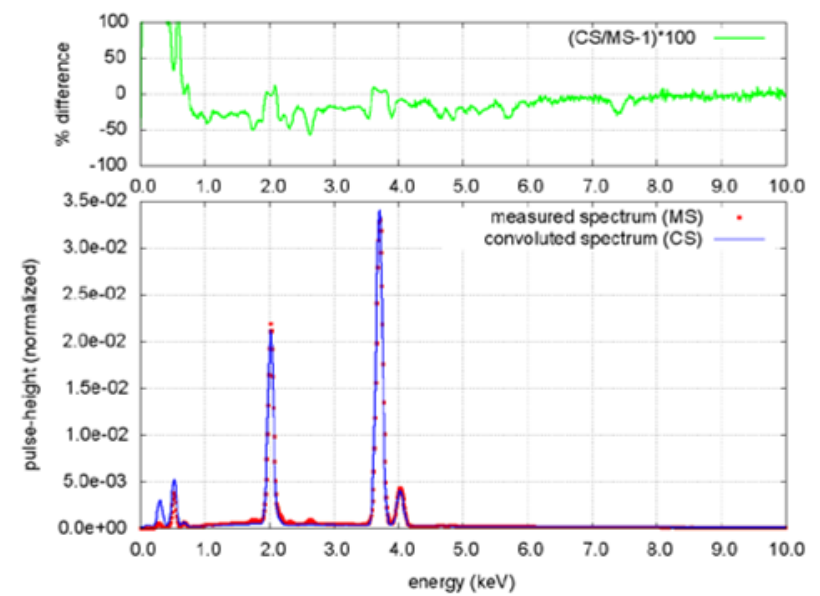

(b) After convolution

Figure 1. Results before and after the convolution process on the apatite $(\mathrm{O}: \mathrm{F}: \mathrm{P}: \mathrm{Ca})$ sample.

\section{Inverse analysis of SEM-EDS spectra}

Traditional SEM-EDS analysis uses properly calibrated standards to identify the elements in an unknown sample. Usually, X-ray energies from the unknown sample shown 
in the EDS spectrum are compared with known characteristic X-ray energy values to determine the presence of an element in a sample. Quantitative results can be obtained from peak heights, i.e., the relative X-ray counts at the characteristic energy levels for the sample constituents. X-ray intensity ratios are calculated by using the sample intensity and the standard specimen intensity for each element present in the unknown sample. The measured intensity ratios are equivalent to the ratios of mass or weight fractions of the constituents in the sample.

A complete $\mathrm{MC}$ simulation of sample interactions covers the scattering of the primary radiation and includes second- and higher-order effects such as the enhancements of fluorescence lines by higher-energy fluorescence or scattered radiation. As a result, it is possible to predict the complete spectral response of an SEM-EDS spectrometer. A significant advantage that the quantification scheme based on MC simulation has over traditional analysis methods is that the simulated spectrum can be compared directly to the experimental data in its entirety, taking into account not only the fluorescence line intensities but also the scattered background of the EDS spectrum. This is coupled with the fact that MC simulations are not limited to first- or second-order approximations or to ideal geometries.

\subsection{Theory}

Our inverse methodology is a multilevel iterative search algorithm that seeks a solution for the parameter vector to minimize the differences between the measured SEMEDS X-ray spectrum and the computed spectrum of the unknown sample. The elements of the parameter vector correspond to the elemental inventories of the sample. This minimization operation is performed over the entire spectrum rather than using only characteristic X-ray peaks. At a high level, the inverse analysis of a measured SEM-EDS spectrum proceeds via the following steps.

1. An initial guess of the elemental composition must be provided and must include all the possible elements within the unknown sample. Effectively, this step constitutes a qualitative analysis of the spectrum.

2. The initial elemental composition is used as input to penORNL to calculate an SEM-EDS spectrum. If the difference between the calculated and measured spectra is less than a prescribed tolerance, the elemental composition is assumed to be optimal and the analysis stops. Since specific elemental inventories have been provided, this step is a quantitative analysis. This step is referred to as an outer iteration.

3. If the convergence criterion in Step 2 is not met, then the elemental inventories are modified via an iterative algorithm to minimize the difference between the calculated and measured spectra. When some prescribed convergence criterion is met, then an updated elemental composition has been determined. Each of these iterations is referred to as an inner iteration.

4. To ensure that the new elemental composition is indeed the best result, this procedure returns to Step 2 to repeat the $\mathrm{MC}$ simulation using the elemental composition produced by Step 3 as the input in Step 2. In other words, a new outer iteration is started.

\subsection{Levenberg-Marquardt}

The minimization problem for the SEM-EDS inverse calculations can be expressed as a sum-of-squared-error minimization with the following objective function:

$$
Y(\alpha)=\sum_{i=1}^{N}\left[X_{i}-C_{i}(\alpha)\right]^{2},
$$

which is minimized with respect to the parameter vector $\alpha . X_{i}$ is the measured spectrum at channel $i$, and $C_{i}$ is the computed spectrum for the given parameter vector $\alpha$ at channel $i$ [see Eq. (4a)]. The problem described in Eq. (5) is a nonlinear least-squares problem and can be solved by the Levenberg-Marquardt nonlinear optimization algorithm [6,7]. This algorithm requires a penORNL calculation for each perturbation made to the elemental weight fractions of the parameter vector and additional calculations for the derivatives (see details in reference 8).

\subsection{Modified algorithm for SEM-EDS}

Although the traditional Levenberg-Marquardt algorithm is a reliable approach, it is not feasible for the scope of this project because it requires multiple penORNL calculations at each inner iteration. Hence a modified version of the search procedure has been developed that utilizes a linearization of the computed solution. In this methodology, the MC line spectrum is decomposed into two components and linearized:

$$
M_{j}\left(\alpha^{k}\right)=B_{j}\left(\alpha^{0}\right)+\sum_{l} \frac{\alpha_{l}^{k}}{\alpha_{l}^{0}} P_{j l}\left(\alpha^{0}\right),
$$

where $B_{j}$ is the background spectrum, $P_{j l}$ is the line spectrum in channel $j$ due to element $l$, and the summation is taken over all elements considered. As noted by the dependence on $\alpha^{0}$, the background spectrum $B_{j}$ and the line spectrum $P_{j l}$ remain constant through all inner iterations. The only quantity that changes is $\alpha_{l}^{k}$, which is the updated inventory of element $l$ at inner iteration $k$. Inherent in this formulation are the following assumptions.

1. The characteristic line intensities of the spectrum are approximately proportional to the amount of each element in the sample (parameter vector). As a result, the characteristic line spectrum can be expressed with a linear dependence on the parameter vector.

2. The background component does not change with the parameter vector.

As a result of the linearization, a modified version of the Levenberg-Marquardt iteration involves a single penORNL calculation at the beginning of each outer iteration. However, the linearization also implies that a globally optimal solution may not be achieved at the end of the search process. Rather, it only provides a best estimate for a given penORNL simulation because the 
background is assumed constant. To correct this problem, we perform another penORNL calculation using the optimal parameter vector from the previous set of inner iterations. This allows updating of the background and characteristic line spectra, after which a new optimal vector is determined using the linearized search process. This multilevel search process is repeated until no further improvement is obtained.

An outline of the multilevel search algorithm is depicted in Figure 2. Starting with the fixed sample, detector/electron beam geometry, and an initial parameter vector (discussed below), the search algorithm performs a penORNL calculation to simulate the SEM-EDS process. Then, the algorithm computes the sum of the squares of the difference between the computed spectrum and the experimental data. If the desired convergence criterion is satisfied, then the calculation is terminated. Otherwise, the current parameter vector and corresponding computed spectrum are used as input for the modified LevenbergMarquardt search process to seek a better solution vector. Another penORNL simulation is initiated using the updated elemental compositions, and the above procedures are repeated until the best solution vector is found.

In this methodology, there are two iteration loops: (1) outer iteration (or step), in which the penORNL calculation is performed, and (2) inner iteration (or just iteration), which is the iteration of the modified Levenberg-Marquardt search process. The characteristic line contribution to the spectrum is updated during Step 2 through the linear dependence on $\alpha^{k}$, whereas the background component is only updated after a penORNL calculation is performed at the start of Step 1. This arrangement might degrade the overall performance for some cases in which the background contribution is significantly higher than the contribution of the characteristic lines. Another problem arises if the characteristic lines of two or more elements are scored in the same channel of the MC line spectrum. To minimize the effects of this issue, the line emission probabilities from the penORNL simulation are provided to the search algorithm in order to make a first order estimate of the intensity of the characteristic lines within the same channel of the MC line spectrum.

The modular code framework MOZAIK-SEM was developed to perform the inverse calculations described above. It is patterned after an existing code MOZAIK, which is a code package that enables automatic geometry optimization for nuclear applications [9]. One of the benefits of this framework is its modularity. For example, penORNL and its auxiliary modules (preprocessor and postprocessor modules) can be replaced with another simulation code without modifying the MOZAIK-SEM framework. Similarly, the modified LevenbergMarquardt methodology could be replaced with another optimization approach. This flexibility allows one to test many options within the same analysis framework.

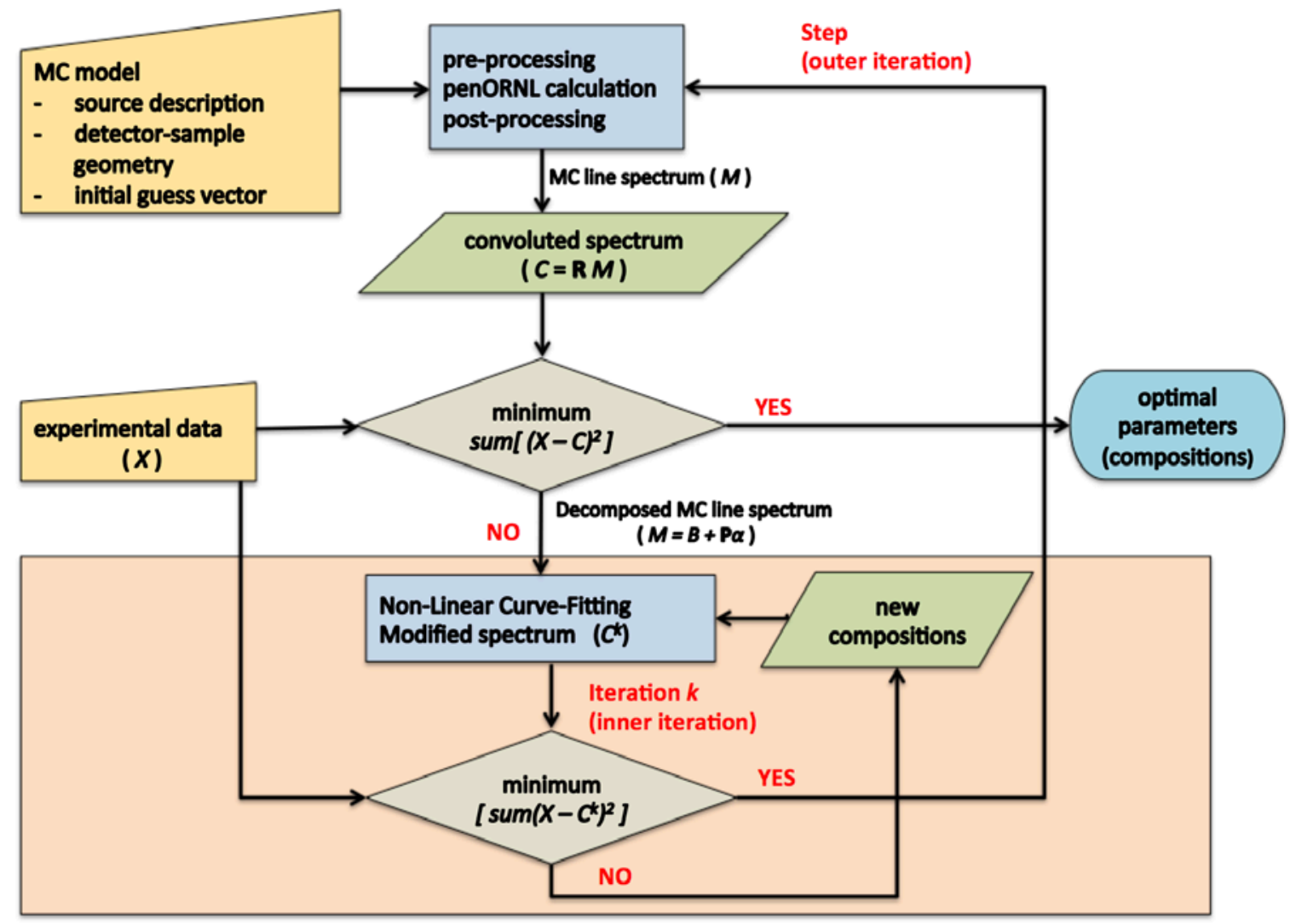

Figure 2. Outline of the iterative search process. 


\section{Initial guess estimation}

Inverse calculations require specification of an initial parameter vector (the elements and their inventories). MOZAIK-SEM does support a user-defined initial parameter vector, although an automatic specification is more useful if no elemental knowledge is available. For this purpose, an initial guess-generation module (MOZAIK-IGM) was developed and integrated into MOZAIK-SEM. This module enables a fully automated search process to construct an initial guess of the parameter vector, which represents both qualitative and quantitative analysis without user insight. It is divided into three submodules, described in the following subsections. The tasks described in the subsections are performed sequentially in the order described.

\subsection{Peak analysis}

The Peak Analysis submodule involves the following tasks:

1. Performs smoothing in the reference spectrum (measured spectrum) using one of several widely used algorithms

2. Analyzes the reference spectrum and marks the possible peak locations

3. Obtains (from the penORNL element database) X-ray line energies close to the peak locations that have been marked

4. Confirms the peak locations if they match any X-ray line energy in the penORNL element database; and

5. Removes from the element list any element whose characteristic X-ray lines do not match any peaks in the reference spectrum

The Peak Analysis submodule uses either exact X-ray line energies or the X-ray line energies within an energy interval (energy of the marked peak $\pm 50 \mathrm{eV}$ ) to confirm the marked peak as a characteristic X-ray.

Figure 3 demonstrates the peak identification performed by the Peak Analysis submodule for the reference spectrum of an Inconel sample. In this case, the reference spectrum was computed by penORNL with $10^{9}$ source particles, which allows the Peak Analysis submodule to skip the smoothing step since noise on the reference spectrum is minimal. The methodology was able to confirm all identified peak locations because each peak matches at least one characteristic X-ray line in the element database. The Peak Analysis submodule provides these results (identified peaks and elements associated with these peaks) as an input to the next submodule within the MOZAIK-IGM module.

\subsection{Spectrum reconstruction}

An algorithm was developed to construct a spectrum from the individual spectra of all the elements identified by the Peak Analysis submodule. A simulated spectrum database has been generated by performing a penORNL calculation for most naturally occurring elements in the periodic table $(Z=5-92)$ and convoluting with the generic detector response function described in section 3 .

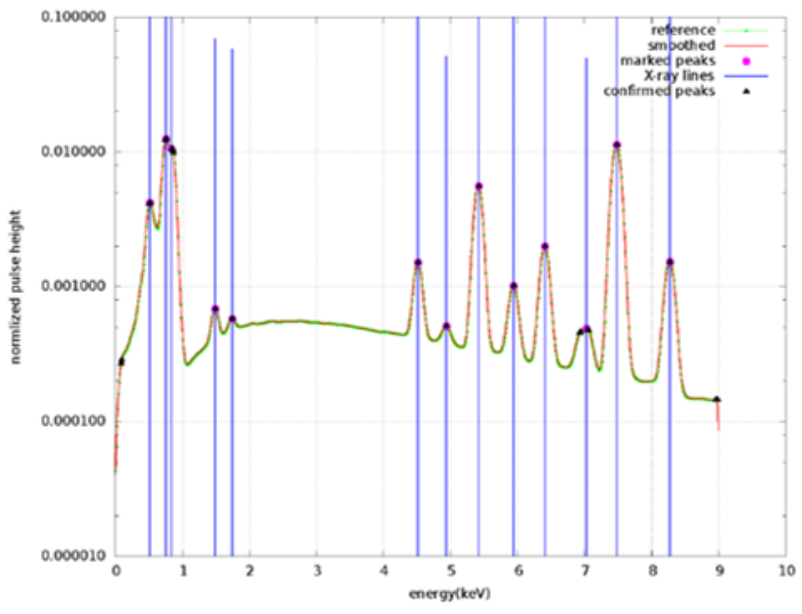

Figure 3. Characteristic X-ray peaks identified and marked by the Peak Analysis submodule for Inconel.

After obtaining the element list from the Peak Analysis submodule, the Spectrum Reconstruction submodule constructs the sample spectrum by multiplying each elemental spectrum from the database by the corresponding elemental weight fraction and adding all these together. Then it passes this constructed spectrum to the next submodule.

One of the deficiencies in this approach is that it does not include the matrix effect whereby one element's characteristic X-ray can excite another element's electrons, which can subsequently produce a characteristic $\mathrm{X}$-ray from the other element. Therefore, this constructed spectrum is preliminary and must be refined by further calculation.

\subsection{Nonlinear least-squares search}

This submodule uses a simple optimization scheme to find optimal weights for the spectrum construction process. The purpose of this step is to minimize the size of the parameter vector (by removing elements whose contribution to the composite spectrum is insignificant) rather than to obtain correct weights. The elemental weights are preliminary at this point (matrix effects have not been considered), and final values are only assigned after a full physics calculation is performed. This optimization in the Initial-Guess Module is necessary because the Peak Analysis submodule might add two or more elements that have characteristic lines matching the same identified peak in the reference spectrum.

\section{Verification and validation}

In this paper, the result of two verification simulations and one validation simulation are presented. In the verification simulations the "experimental data" are actually the results of penORNL simulations using the exact weight fractions of a sample with different random number seeds and smaller MC uncertainties than the MOZAIK-SEM inverse simulations. In other words, the verification simulations are primarily a test of our inverse analysis methodology implemented in MOZAIK-SEM. The validation simulation uses actual experimentally measured data 
provide by NIST, which tests our inverse analysis methodology in MOZAIK-SEM and the PENELOPE physics in penORNL.

\subsection{Verification with $\mathrm{U}_{3} \mathrm{O}_{8}$}

For this verification simulation, the sample consists of a cube of $\mathrm{U}_{3} \mathrm{O}_{8}\left(6.83 \mathrm{~g} / \mathrm{cm}^{3}\right)$ measuring $50 \mu \mathrm{m}$ on all sides. It was placed $2 \mathrm{~cm}$ away from the $25 \mathrm{keV}$ electron beam source, and the X-ray spectrum was tallied for a generic $\mathrm{Si}(\mathrm{Li})$ detector. Energy bin widths were set to $10 \mathrm{eV}$ to match the typical channel width in SEM-EDS detectors. Since this is a verification calculation, a reference spectrum was created by penORNL simulating 1 billion source particles (electrons) to reduce the statistical noise in the X-ray spectra.

One difficult aspect of this spectrum is that the sample contains a light element, oxygen. Traditional quantitative analysis has had difficulty correctly identifying the weight fractions of light elements. On the other hand, there are few overlapping peaks in the X-ray spectrum and only $\mathrm{U}$ and $\mathrm{O}$ are included in the initial guess of the elemental composition (parameter vector).

The initial parameter vector for this verification simulation contained a guess that half of the unknown sample was $\mathrm{U}$ and the other half $\mathrm{O}$, by weight. Figure 4 compares the reference $\mathrm{U}_{3} \mathrm{O}_{8}$ spectrum and the spectrum of the composition MOZAIK-SEM calculated. Also included in Figure 4 is a plot of the percent difference between the reference and MOZAIK-SEM spectra. There is very little observable difference between the two spectra in Figure 4, but the largest percent difference in the spectra occurs in the tails of photopeak at $3 \mathrm{keV}$. The actual composition of the $\mathrm{U}_{3} \mathrm{O}_{8}$ sample (weight fractions) is shown in Table 2, which also includes the elemental composition calculated by MOZAIK-SEM along with the percent difference between the calculated and reference elemental composition. The percent difference between the reference and MOZAIK-SEM O weight fraction is nearly $1.2 \%$, but the absolute difference for both elements' weight fraction is less than 0.01 .

\subsection{Verification with K497}

This verification simulation is nearly identical to the previous simulation. However, the composition of the sample has been changed from $\mathrm{U}_{3} \mathrm{O}_{8}$ to that of a NIST standard glass, K497 $\left(1.79 \mathrm{~g} / \mathrm{cm}^{3}\right)$.

This verification simulation is interesting because K497 is composed of 12 elements, and some of these elements have weight fractions significantly less than others. Moreover, several characteristic X-ray lines of the 12 elements overlap, contributing to the difficulty of this problem.

Table 3 presents the weight fractions of each element estimated by MOZAIK-SEM. The results of this verification test are a mix of positive and negative observations:

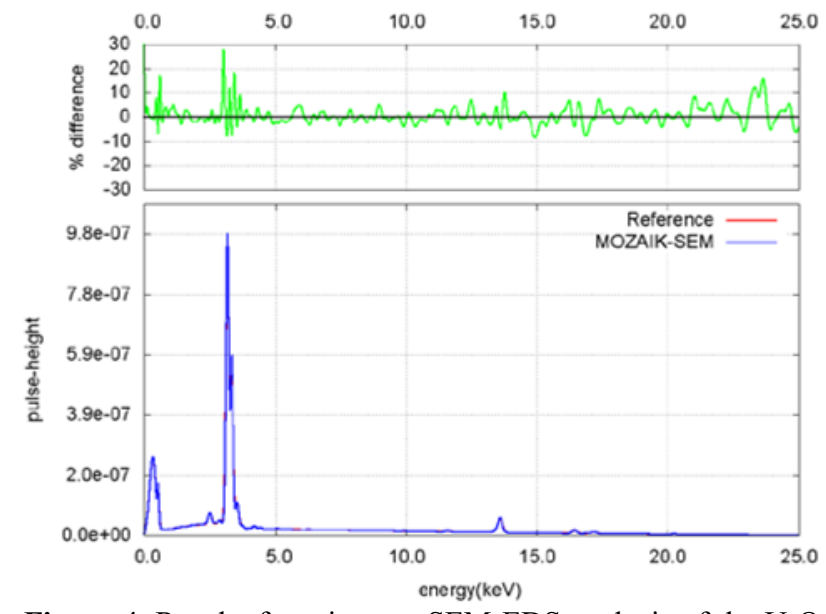

Figure 4. Results from inverse SEM-EDS analysis of the $\mathrm{U}_{3} \mathrm{O}_{8}$ sample. Inverse analysis was accomplished in 4 outer iterations.

Table 2. Elemental composition of the $\mathrm{U}_{3} \mathrm{O}_{8}$ sample $\left(10^{8}\right.$ source particles in each penORNL simulation within MOZAIK-SEM)

\begin{tabular}{cccc}
\hline Element & Reference & MOZAIK-SEM & \% diff \\
\hline $\mathrm{U}$ & 0.848001 & 0.84980 & 0.2122 \\
$\mathrm{O}$ & 0.151999 & 0.15020 & -1.1836 \\
\hline
\end{tabular}

1. All elements with weight fractions greater than $0.01(\mathrm{O}$, $\mathrm{Mg}, \mathrm{Al}, \mathrm{P}$ ) were estimated with relative error less than 3\%; Fe also was estimated very well. The oxygen prediction with less than $1 \%$ error is especially significant.

2. For trace elements (weight fractions $<0.01$ ), the estimated weight fractions contained larger relative error (even double or triple digits). The element B was completely discarded. These errors are at least partly related to $\mathrm{MC}$ uncertainties, as the characteristic X-ray peaks of these elements were statistically indistinguishable from the background portion of the simulated spectrum. 3. Unusual behavior was exhibited by $\mathrm{P}$ and $\mathrm{Zr}$. These elements have characteristic X-ray line energies that are very close to each other and their peaks overlap. The search process estimated the total weight fraction for these two elements with a $0.65 \%$ difference (reference, $0.3318+$ $0.004=0.3358 ;$ MOZAIK-SEM, $0.3229+0.01507=$ 0.33797 ) but could not resolve the contribution of each element individually - the $\mathrm{Zr}$ weight fraction is overestimated, and the $\mathrm{P}$ weight fraction is (slightly) underestimated.

4. The absolute errors for all elements except $\mathrm{Zr}$ are less than $1 \%$ of the total assay. $\mathrm{Zr}$ is actually present as a trace quantity (fraction 0.004) but is predicted to have small, but non-trace, assay fraction (1.5\%).

Figure 5 presents the comparison of the reference spectrum and the calculated inverse spectrum. The plot shown at the top of Figure 5 compares the spectra over the full energy range, and the plot at the bottom presents the same comparison over the energy range 0.0 to $5.0 \mathrm{keV}$. Discrepancies in the spectrum plots themselves are indistinguishable, although the difference plots indicate slight errors, especially around peak energies. 
Table 3. Elemental composition of the K497 sample $\left(10^{8}\right.$ source particles in each penORNL simulation within MOZAIK-SEM).

\begin{tabular}{lllr}
\hline Element & Reference & $\begin{array}{l}\text { MOZAIK-SEM } \\
( \pm \text { MC error })\end{array}$ & \% diff \\
\hline $\mathrm{B}$ & 0.0005 & $0.00000( \pm 0.00000)$ & --- \\
$\mathrm{O}$ & 0.5245 & $0.52648( \pm 0.00035)$ & 0.38 \\
$\mathrm{Mg}$ & 0.0521 & $0.05129( \pm 0.00042)$ & -1.56 \\
$\mathrm{Al}$ & 0.0578 & $0.05679( \pm 0.00036)$ & -1.74 \\
$\mathrm{Si}$ & 0.0013 & $0.00003( \pm 0.01154)$ & -97.66 \\
$\mathrm{P}$ & 0.3318 & $0.32290( \pm 0.00035)$ & -2.68 \\
$\mathrm{Ti}$ & 0.0021 & $0.00230( \pm 0.00119)$ & 9.51 \\
$\mathrm{Fe}$ & 0.0024 & $0.00243( \pm 0.00112)$ & 1.22 \\
$\mathrm{Zr}$ & 0.0040 & $0.01507( \pm 0.00007)$ & 276.74 \\
$\mathrm{Ce}$ & 0.0062 & $0.00549( \pm 0.00067)$ & -11.43 \\
$\mathrm{Ta}$ & 0.0080 & $0.00933( \pm 0.00101)$ & 16.56 \\
$\mathrm{~Pb}$ & 0.0092 & $0.00789( \pm 0.00142)$ & -14.19 \\
\hline
\end{tabular}
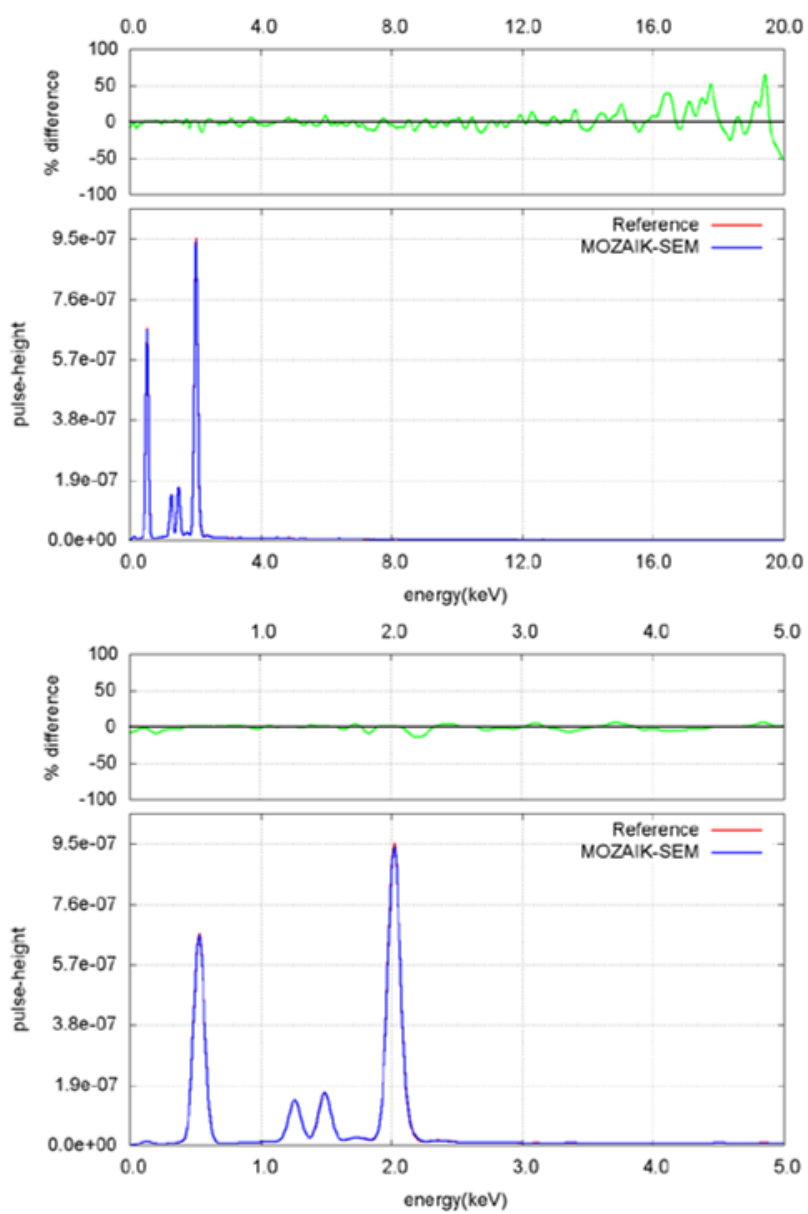

Figure 5. Results from inverse SEM-EDS analysis of the K497 glass sample. Inverse analysis was accomplished in 22 outer iterations.

\subsection{Summary of Verification}

All the results from the verification study are summarized below.

1. The methodology within MOZAIK-SEM is a feasible tool for SEM-EDS inverse calculations.

2. The $\mathrm{MC}$ errors in the results from penORNL simulations limit the performance of MOZAIK-SEM for trace elements. Better results can be obtained for elements with very low weight fractions by performing longer penORNL calculations that produce smaller MC errors for the background portion of the spectrum and legitimate small photopeaks within the spectrum.

3. In these examples, MOZAIK-SEM estimated the weight fraction of the elements correctly if the fraction was larger than 0.01 . The $\mathrm{MC}$ errors for large photopeaks were usually on the order of 0.01 or less. In almost every case, the predicted elemental composition was within $2 \%$ of the actual assay value.

4. MOZAIK-SEM resolved many overlaps within the spectrum; however, it demonstrated some problems with the following challenging cases.

A. Overlaps in the MC line spectrum. This occurs when two or more elements' characteristic X-ray line energies are so close that the $\mathrm{MC}$ code scores these lines in the same energy bin of the tally.

B. Overlaps in the convoluted spectrum. This occurs when two or more elements' characteristic X-ray line energies are different enough that they appear in different energy bins of the MC line spectrum. However, when the MC line spectrum is convoluted with the detector response function, the characteristic X-ray energies are close enough that only a single photopeak appears in the convoluted spectrum.

\subsection{Validaton with fluorapatite}

In this validation calculation a measured spectrum provided by NIST for a material standard was used to evaluate the performance of MOZAIK-SEM. The material to be considered is the mineral fluorapatite $\mathrm{Ca}_{10}\left(\mathrm{PO}_{4}\right)_{6} \mathrm{~F}_{2}$, whose elemental weight fractions are given in the second column of Table $4\left(3.15 \mathrm{~g} / \mathrm{cm}^{3}\right)$. Using an initial guess distributed uniformly among the elements produces the estimated weight fractions given in the third column, which contain noticeable discrepancies. However, all elements are estimated to within 5\% absolute error.

Table 4. Elemental composition of the apatite sample $\left(10^{8}\right.$ source particles in each penORNL simulation within MOZAIK-SEM).

\begin{tabular}{lccr}
\hline Element & Reference & MOZAIK-SEM & \% diff \\
\hline $\mathrm{O}$ & 0.38071 & 0.30041 & -21.092 \\
$\mathrm{~F}$ & 0.03767 & 0.03506 & -6.928 \\
$\mathrm{P}$ & 0.18426 & 0.21519 & 16.786 \\
$\mathrm{Ca}$ & 0.39736 & 0.44937 & 13.089 \\
\hline
\end{tabular}

The following are several possible issues that could have contributed to the error in predicted weight fractions. 1. As shown in Figure 6, the peak centroid energies for the two spectra are slightly different, which means the energy bin structure in the penORNL simulation may be different from that of the reference spectrum. The energy bin structure of the simulation is based on data provided in the NIST data file. It is possible that the detector was not calibrated immediately before the measurement was taken, so the recorded bin structure may not be correct. Additionally, the relationship between channel number 
and X-ray energy may not be linear, which appears to be the assumption applied by NIST.

2. All our calculations used a generic detector response function that works well for the verification calculations (see section 3). A response function tailored to the particular detector, and possibly the SEM itself, might improve the results.

3. It is possible that the electron source specification in our computational model is not consistent with the actual SEM used because the backgrounds from both spectra have different slopes (Figure 7). The electron spectrum was reported as a single energy, which is likely not correct. That energy is probably the mean electron energy, but no information about the energy distribution is available.

4. Figure 7 compares the measured spectrum with a computed spectrum using the known elemental composition. It indicates that the measured spectrum exhibits some peaks that are not shown in the computed spectrum. The energies of these peaks do not match the characteristic X-ray line energies of the elements in the apatite sample, so they do not appear in the computed penORNL spectrum.

A. If these "extra" peaks do not belong to the elements in the sample, they might be sum peaks, which our generic response function does not account for.

B. Another possibility is that these are characteristic Xrays of some contaminant element (in the sample itself or in the sample environment) that is not in our computational model.

C. The final possibility is that there is an unidentified error (or errors) in the penORNL physics model or in the atomic data.

For all these possible reasons, MOZAIK-SEM could not estimate the weight fraction for this sample more accurately. At this time, not enough information is available to rule out any of these explanations. Because the sample represents an archived spectrum made several years ago, it is not likely that additional information about the measured spectrum can be obtained.

\section{Conclusions and future work}

The overall conclusion of this work is that MOZAIK-SEM is a promising method for performing inverse analysis of $\mathrm{X}$-ray spectra generated within a SEM, but additional work is needed to refine and validate this methodology. The methodology implements a two-tiered strategy: an outer iteration, which requires a full $\mathrm{MC}$ calculation, and an inner iteration, which refines elemental estimates quickly. The latter uses a modified Levenberg-Marquardt iteration to solve a linearized problem, which reduces the inverse analysis into two pieces, the photopeaks and the background. This approach has the benefit of reducing the number of $\mathrm{MC}$ simulations required, although the background portion of the spectrum and the matrix effects on the spectrum are only updated after each outer iteration.

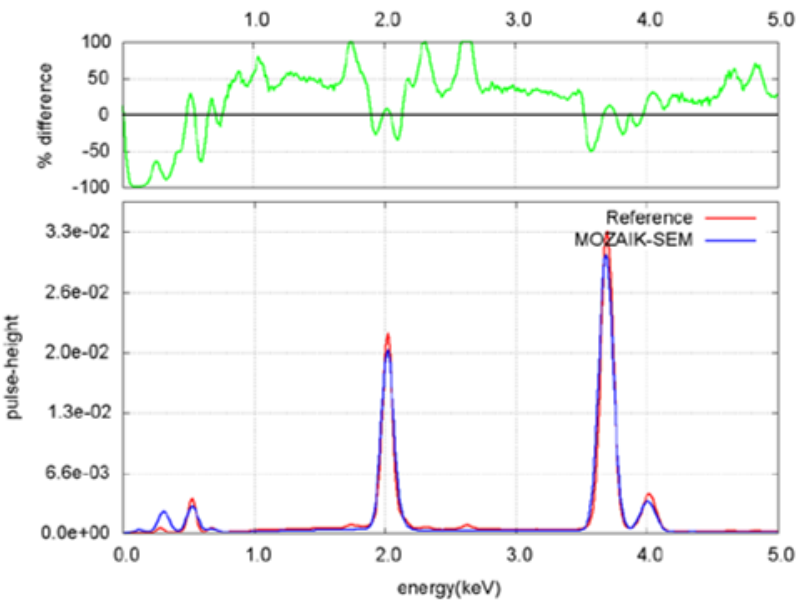

Figure 6. Results from inverse SEM-EDS analysis of the apatite sample. Reference is the standard's spectrum from NIST.

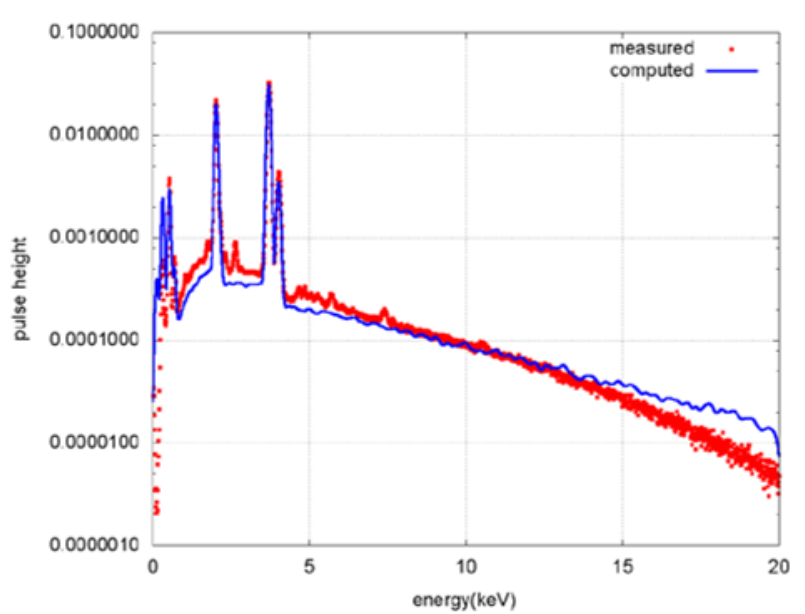

Figure 7. Comparison of measured and calculated spectra for the apatite sample. The measurement was supplied by NIST and the computed spectrum by penORNL using the actual elemental composition.

Verification simulations with MOZAIK-SEM revealed that this methodology can successfully identify the weight fractions of low-Z elements such as oxygen and fluorine. However, like other quantitative analysis methods, this method can also suffer when X-ray line energies for multiple elements are very close together. A problem unique to this method is in distinguishing small photopeaks due to trace elements from MC statistical noise in the X-ray background; the problem occurs when the background MC uncertainty is of the same order of magnitude as the weight fraction of a trace element. Simulating more particle histories during the inverse analysis helps reduce this issue, but that increases the computational burden in execution time or additional processors. Finally, the validation simulation with MOZAIK-SEM was less successful than the verification simulations, at least in part due to vaguely defined conditions of the experimental measurement. A number of prominent differences exist between the spectrum measured by NIST and the spectrum simulated by penORNL. In the end, no source of these differences can be absolutely identified, so it is impossible to state if these differences are due to the measurements, MOZAIK-SEM 
methodology, penORNL physics, or the interaction data it uses.

For the MOZAIK-SEM methodology to be improved, two issues need to be addressed. First, a better method to deal with X-ray line energies that are very close together should be investigated. The method as it exists now works fairly well, but there is clearly room for improvement. Second, and more importantly, additional validation simulations should be performed, but this should be done as part of a much closer collaboration between the experimentalist and modeler. For the validation effort to be successful, future measurements should include (1) an energy calibration of the SEM-EDS detector, (2) an energy calibration of the SEM electron source, and (3) greater details of the geometry, including the sample, detector, and, of lesser importance, the internal configuration of the SEM sample chamber.

\section{Acknowledgments}

This work was performed with funds provided by the US DOE through the Nonproliferation Enabling Capabilities program within the Office of Defense Nuclear Nonproliferation Research and Development.

The authors of this report gratefully acknowledge the assistance provided by NIST, namely, John Henry Scott, Nicholas W. M. Ritchie, and Dale E. Newbury.

Finally, the authors would also like to thank Francesc Salvat for his assistance and insight into PENELOPE.

\section{References}

1. T. M. Miller, B. W. Patton, and C. F. Weber, Trans. of the Amer. Nuc. Soc. 110, 497 (2014)

2. F. Salvat, J. M. Fernandez-Varea, and J. Sempau, PENELOPE, a code system for Monte Carlo simulation of electron and photon transport, Universitat de Barcelona (2011)

3. F. Salvat et al., Microchimica Acta 155, 67 (2006)

4. MCNP6 Development Team, MCNP6 User's Manual Version 1.0, Los Alamos National Laboratory, LACP-13-00634, Rev. 0 (2013)

5. K. B. Bekar et al., ANS MC2015 - Joint International Conference on Mathematics and Computation $(M \& C)$, Supercomputing in Nuclear Applications (SNA) and the Monte Carlo (MC) Method (2015)

6. K. Levenberg, Quart. of App. Math. 2, 164 (1944)

7. D. W. Marquardt, J. Soc. Ind. and App. Math. 11, 431 (1963)

8. K. B. Bekar et al., Rapid Evaluation of Particle Properties Using Inverse SEM Simulations, Oak Ridge National Laboratory, ORNL/TM-2015/779 (2016)

9. K. B. Bekar, Modular Optimization Code Package: MOZAIK, PhD dissertation, The Pennsylvania State University, University Park, Pennsylvania (2008) 